\title{
Cuando la población migrante desmiente los discursos dominantes: autorretratos lingüístico-culturales de jóvenes hablantes bilingües del español en Suiza*
}

\author{
Víctor Fernández-Mallat ${ }^{1}$ \\ Georgetown University (Estados Unidos)
}

\section{Resumen}

En los discursos político y popular suizos, las poblaciones migrantes suelen ser concebidas como un sector demográfico que se integra difícilmente a la llamada sociedad local, tanto lingüística como culturalmente. En este estudio, con base en los autorretratos lingüístico-culturales de siete hablantes bilingües del español, muestro que tales discursos no reflejan las maneras en las que estas personas conciben sus prácticas idiomático-culturales cotidianas. Más bien, las prácticas que describen ponen de relieve que han desarrollado habilidades bilingües y biculturales sin par que les permiten, entre otras cosas, dar relevancia a ciertos aspectos de los repertorios lingüísticos e identidades culturales propios en función de elementos contextuales. Estas observaciones se alinean con estudios previos que, con base en datos censales y discursivos, muestran que el

\section{Abstract}

In Swiss political and popular discourses, migrant populations are often depicted as unable to easily integrate to the so-called local society, both linguistically and culturally. In this study, drawing from the linguistic and cultural self-portraits of seven bilingual speakers of Spanish, I show that such discourses do not reflect the ways in which these populations conceive of their daily language and cultural practices. Rather, the practices they describe show that they have developed unparalleled bilingual and bicultural skills that, among other things, allow them to give relevance to certain aspects of their linguistic repertoires and cultural identities in accordance with contextual elements. These observations build on prior research that, based on census and discursive data, show that the development of such skills is not limited to second generation

\footnotetext{
* When migrants debunk dominant discourses: the linguistic and cultural self-portraits of young bilingual speakers of Spanish in Switzerland

1 Doctor en lingüística hispánica por la Universidad de Montreal (Canadá). Profesor del Departamento de español y portugués de la Universidad de Georgetown (Estados Unidos). e-mail: victor.orlando.fernandez@georgetown.edu
} 
desarrollo de tales habilidades no se limita a la población migrante de la segunda generación; también se observan destrezas similares entre migrantes de la primera generación.

Palabras clave: Sociolingüística, análisis del discurso, identidades lingüísticas y culturales, ideologías lingüísticas, migrantes hispanohablantes en Suiza. migrants; similar skills are also observed among first generation migrants.

Keywords: Sociolinguistics, discourse analysis, linguistic and cultural identities, language ideologies, Spanish-speaking migrants in Switzerland.

\section{Introducción}

A raíz de los movimientos poblacionales transnacionales del último tercio del siglo XX y comienzos del XXI, se ha extendido en los llamados países receptores de migrantes un discurso eminentemente asimilacionista que magnifica el aprendizaje de lenguas y valores locales o nacionales como la clave de la integración cívica de la población migrante (Fernández-Suárez, 2017:118). Uno de los principales problemas de este tipo de discursos es que contribuye a la emergencia de una imagen de los migrantes como un sector demográfico que no se integra ni lingüística ni culturalmente a la población percibida como local. García (1993:78), por ejemplo, advierte que, en los Estados Unidos, como consecuencia de tales discursos, sobresale una imagen de los latinos como una población extranjera que no habla inglés, independientemente de que efectivamente hablen o no esta lengua y de que hayan nacido o no en ese país. Por su parte, Johnstone (2008:150) nota que, en Alemania, la población migrante perteneciente a la segunda generación es comúnmente tachada de "extranjeros" en el discurso dominante, a pesar de haber nacido y haberse escolarizado y socializado en el país. Siguiendo los conceptos de iconización y ocultamiento ${ }^{2}$ de Irvine y Gal (2000), no solo puede observarse en los países que reciben migrantes una clara propensión a convertir a estas personas y sus prácticas lingüístico-culturales en índices que evocan la falta de integración, sino también una marcada tendencia a ocultar la diversidad inherente a la población migrante en lo tocante a estas prácticas. En otras palabras, existe una proclividad a invisibilizar el hecho de que, dentro de esta población, existen personas con distintos grados de conocimiento e integración de la(s) lengua(s) y los valores del lugar en el que se han instalado. Como consecuencia, en vez de entenderse los idiomas y las cualidades de los migrantes como habilidades, se conciben como un pro-

2 Los términos en español provienen del trabajo de Sánchez (2016). En la obra de Irvine y Gal (2000), que está en inglés, aparecen como iconization y erasure. 
blema que debe solucionarse. En efecto, tal y como lo señala Fernández-Suárez (2017:119), la respuesta de muchas de las naciones en las que se asientan migrantes ante la popularización de este discurso asimilacionista ha consistido en implementar políticas migratorias que establecen una sujeción entre el grado de integración cívica y la emisión o renovación de los permisos de residencia y/o trabajo de la población migrante. Como advierte la autora (p. 119), este nivel de integración suele medirse mediante pruebas estandarizadas de lengua y cultura locales.

Suiza, el país en el que centro el análisis del presente trabajo de investigación, no es ajena ni a este discurso ni a las políticas que se ponen en funcionamiento como consecuencia de su propagación. En efecto, según Flubacher (2013:173), en lo tocante al país helvético, los dos hitos discursivos más imponentes de la ideología asimilacionista son la moción de Bircher (1998) y la consiguiente publicación de la Ley Federal de Extranjería ${ }^{3}$ (2005). Como advierte la autora (Flubacher, 2013:173), la propuesta de Bircher (1998) no solo tuvo como consecuencia que saliera a relucir a nivel nacional la idea de que la clave de la integración es el aprendizaje de una de las lenguas nacionales ${ }^{4}$; también contribuyó a que se propagara la idea de que tanto los migrantes como sus hijos no se integran a la sociedad suiza de manera adecuada y de que esto es un problema que podría incluso llegar a comprometer la seguridad nacional. Tal y como lo señalan Flubacher (2013:174) y Yeung (2016:731), la Ley Federal de Extranjería (2005) tuvo como efecto que las ideas de la propuesta de Bircher (1998) se volvieran jurídicamente vinculantes. En efecto, el quincuagésimo cuarto artículo de dicha ley establece que la emisión de permisos de residencia para extranjeros depende de su grado de integración a la sociedad suiza. Este se mide sobre la base de su nivel de conocimiento de una de las lenguas locales. Como Suiza no tiene derecho del suelo (o ius soli), la ley también se aplica a los hijos de los migrantes e incluso a sus nietos, a los que se refiere comúnmente como "secondos" y "terceros" (Wessendorf, 2013:99), a pesar de que hayan nacido en Suiza y se hayan escolarizado en una de las llamadas lenguas nacionales de manera obligatoria. Así pues, es costumbre que personas nacidas y formadas en Suiza deban pasar pruebas de conocimientos lingüísticos y culturales para validar sus permisos de residencia o a fin de naturalizarse. En suma, como se puede ver, el discurso predominante ha contribuido a que se propague una imagen de los migrantes como una población que se integra difícilmente a la sociedad suiza, tanto lingüística como culturalmente. Asimismo,

3 Esta ley entró en vigor en el 2008.

4 En Suiza hay cuatro lenguas nacionales: el alemán, el francés, el italiano y el romanche. Solo las tres primeras son de uso oficial en el parlamento. 
ha contribuido a que se invisibilice el hecho de que esta población constituye una comunidad diversa compuesta por migrantes de primera, segunda y tercera generaciones con distintos niveles de integración lingüístico-cultural. Como consecuencia, a pesar de que, por motivos de capitalización económica, tanto el multilingüismo como el multiculturalismo son vistos en el país helvético como una riqueza (Duchêne y Del Percio, 2014:76), las lenguas no nacionales - entre las que destacan el serbocroata, el albanés, el portugués y el español, en ese orden (Lüdi y Werlen, 2005:11) - y los valores de la población que habla estas lenguas debido a su procedencia o al origen de sus progenitores son entendidos como un problema que requiere ser solucionado.

Aun así, de acuerdo con datos censales del 2014, en Suiza, más de $90 \%$ de la población migrante de primera y segunda generaciones ha incorporado la lengua oficial de la región de asentamiento (i.e. el alemán, el francés o el italiano) a su repertorio lingüístico nativo y la usan regularmente (de Flaugergues, 2016, pp.14-16). Por otra parte, en un estudio de orientación cualitativa con base en la autoidentificación lingüístico-cultural de migrantes hispanohablantes de primera generación que han elegido domicilio en la ciudad francófona de Lausana, Diez del Corral Areta (por aparecer) comprueba que estas personas efectivamente han incorporado la lengua oficial del lugar de asentamiento al repertorio lingüístico adquirido previamente. Específicamente, nueve de las diez personas entrevistadas por la autora se describen como bilingües en español y francés. Asimismo, Diez del Corral Areta (por aparecer) constata que, si bien solo dos de las personas entrevistadas afirman haber incorporado plenamente la identidad cultural suiza a la identidad cultural adquirida antes de instalarse en el país helvético, todas presentan una fuerte integración social. En otras palabras, tanto los datos censales que presenta de Flaugergues (2016) como los datos cualitativos que expone Diez del Corral Areta (por aparecer) ponen de relieve que el discurso según el cual los migrantes no se integran ni lingüística ni culturalmente a la sociedad suiza no refleja la realidad.

Aunque los datos censales presentados por de Flaugergues (2016, pp.14-16) revelan que, entre la segunda generación de migrantes la integración lingüística es aún mayor que entre la población migrante de primera generación, es menor el conocimiento sobre la manera en la que este sector demográfico de la población suiza concibe discursivamente sus propios perfiles lingüísticos. Igualmente, poco se sabe acerca del modo en el que estas personas se autoidentifican culturalmente. En el presente trabajo, me propongo compensar esta escasez de conocimientos mediante el análisis de una serie de interacciones 
conversacionales grabadas en las que siete personas de la segunda generación de migrantes hispanohablantes en Suiza se representan, tanto en lo que concierne a sus perfiles lingüísticos como en lo referente a sus afiliaciones culturales. Así pues, estos autorretratos lingüístico-culturales no solo me ayudan a determinar con base en la percepción de estas personas cuáles son los recursos lingüísticos y rasgos culturales que las caracterizan; también me permiten evaluar su grado de integración a la sociedad nacional a través de sus ojos.

Dar tribuna a estas voces es importante, pues, como señala Johnstone (2008:129), les da agencia a sectores de la población cuyas voces no suelen escucharse y que, por ende, comúnmente carecen de un papel moldeador del mundo. Además, como señalan autores como Hymes (1996) y Blommaert (2005), hacer que las voces de estos colectivos de personas se escuchen permite entender las voces dominantes en términos de políticas e ideologías lingüísticas así como su efecto en estas poblaciones minorizadas. En el caso que me ocupa, esta tribuna les permite a estos siete miembros de la segunda generación de migrantes hispanohablantes participar en conversaciones en las que cuestionan y mantienen a distancia (o no) los estereotipos que circulan en Suiza sobre sus personas. De igual modo, les permite establecer elementos distintivos sobre sus identidades lingüístico-culturales.

Siguiendo a autores como Grosjean (2008) y Schwartz y Unger (2010), abordo el tema desde un enfoque teórico basado en la idea de que es la autoidentificación contextualizada de las personas la que tiene validez al describir sus perfiles culturales. Si bien estos autores aplican inicialmente esta idea a la filiación cultural de personas en contextos biculturales, basándome en Diez del Corral Areta (por aparecer), propongo que este discernimiento puede aplicarse también a la manera en la que las personas se identifican lingüísticamente a sí mismas en contextos bilingües o multilingües. Este enfoque es adecuado para mi trabajo porque me interesa explorar la manera en la que los siete miembros de la segunda generación de migrantes hispanohablantes cuyos autorretratos analizo se alinean o no con las ideas normalizadas que circulan en Suiza sobre su nivel de integración lingüístico-cultural.

En la siguiente sección, describo el estudio empírico. En la sección subsiguiente, presento los hallazgos emergentes de mi análisis. En la sección final, discuto acerca de las contribuciones teóricas y las implicaciones prácticas de mis observaciones. 


\section{El estudio}

Mi estudio se basa en 14 meses de trabajo etnográfico y de investigación participativa en dos instituciones suizas de enseñanza superior. Una de las razones principales por las que decidí llevar a cabo la presente investigación en tales establecimientos es el hecho de que sus programas de español cuentan con un número considerable de personas de la segunda generación de migrantes hispanohablantes en Suiza. Como muchos de ellos mismos me comunicaron, se matricularon en el programa de español de su institución por un interés marcado en (re)conectar con sus raíces y/o (re)descubrirlas. Debido a esa voluntad de darle (mayor) relevancia al elemento hispano o latino de sus identidades lingüístico-culturales en detrimento (o no) de componentes suizos de sus personas, considero que observar la manera en la que se perciben a sí mismas se adecúa al propósito de este estudio de matizar, cuestionar y/o rechazar la idea de que la población migrante es un conjunto homogéneo de personas que se integra con dificultad a la sociedad suiza. En efecto, debido a esta orientación hacia la lengua y cultura presente en sus círculos familiares e íntimos o lugares de procedencia, desde el punto de vista del discurso que se ha convencionalizado en Suiza, podrían ser percibidos como ejemplo de personas que no se integran a la llamada sociedad local. Otra razón por la cual decidí realizar mi investigación en estos dos establecimientos es porque, a lo largo de mi trabajo etnográfico, no me limité a observar a los estudiantes; también tuve la oportunidad de participar en numerosas actividades que me permitieron crear vínculos estrechos con las siete personas que terminaron participando en mi estudio (p. ej., talleres y seminarios que yo animé y eventos sociales que ellos mismos organizaron). Con el paso del tiempo, estas personas se dieron cuenta de que podían hablar conmigo (más) libremente. Poco a poco, comenzaron a participarme de sus opiniones y experiencias que, con su consentimiento, grabé en diferentes ocasiones. En una de estas, les pedí específicamente que se describieran a sí mismas lingüística y culturalmente. Con naturalidad, tal vez debido a la relevancia del tema en Suiza, la cuestión de ser hablante de una u otra lengua (el español o una de las lenguas locales) y el asunto de la prominencia de una u otra identidad (la hispana o latina o la suiza) constituyeron el foco de nuestra conversación.

En lo que se refiere al perfil de las personas cuyos autorretratos lingüístico-culturales sirven de base para mi análisis, como se puede ver en la Tabla 1 de abajo, son bastante diversos. Algunos, como Alberto, Nelly, Tania y Silvia ${ }^{5}$, han nacido y crecido en Suiza; otros,

5 Estos nombres son ficticios. 
como Ana, Paco y Samuel, han nacido en países de habla hispana y crecido en Suiza. Así y todo, siguiendo a Rumbaut (2004), considero que, por haber vivido una parte importante de su periodo formativo en Suiza, estos últimos también son parte de la llamada segunda generación de migrantes. Paco y Samuel llegaron a Suiza a los 12 años; Ana llegó a los 13.

Tabla 1: Perfil de las personas que participaron en el estudio

\begin{tabular}{cccccc}
\hline Nombre & Edad & Género & $\begin{array}{c}\text { Lugar de } \\
\text { nacimiento }\end{array}$ & $\begin{array}{c}\text { Origen de } \\
\text { la madre }\end{array}$ & $\begin{array}{c}\text { Origen } \\
\text { del padre }\end{array}$ \\
\hline Alberto & 25 años & Masculino & Suiza & Suiza & España \\
Ana & 26 años & Femenino & Bolivia & Suiza & Bolivia \\
Nelly & 24 años & Femenino & Suiza & Colombia & Chile \\
Paco & 23 años & Masculino & Colombia & Colombia & Suiza \\
Tania & 23 años & Femenino & Suiza & Suiza & España \\
Silvia & 23 años & Femenino & Suiza & España & España \\
Samuel & 25 años & Masculino & Colombia & Colombia & Colombia \\
\hline
\end{tabular}

De igual manera, como se puede ver, Nelly, Silvia y Samuel tienen progenitores provenientes exclusivamente de países hispanohablantes. En cambio, Alberto, Ana, Paco y Tania son los descendientes de parejas mixtas en las que uno de los progenitores proviene de un país de habla hispana y el otro es originario de Suiza. Toda esta información es relevante porque, al analizar la manera en la que estas personas se perciben a sí mismas lingüística y culturalmente, me permite determinar si factores como su lugar de nacimiento y la procedencia de sus progenitores tienen cierta incidencia en la manera en la que se piensan.

Analizo los autorretratos lingüístico-culturales de Alberto, Ana, Nelly, Paco, Tania, Silvia y Samuel con un enfoque basado en el discurso. Siguiendo a Liebscher y Dailey-0'Cain (2009), aplico tres niveles de análisis. El primero se basa en el contenido; es decir, se apoya en lo que dicen las personas. En el segundo nivel, me intereso por la función que ciertas palabras como mucho y definitivamente pueden adquirir como mecanismos de acentuación o atenuación de lo que las personas dicen. En el tercer nivel de análisis, tengo en cuenta herramientas discursivas como la risa, las pausas y los cambios de tono o intensidad y el papel que juegan en matizar el contenido de los enunciados. 


\section{Resultados emergentes del análisis}

En lo tocante a la autoidentificación lingüística, en función de las respuestas que las personas que participaron en mi estudio dieron a mis interrogantes, reconocí dos grupos: el de las personas que se describen como más hablantes de una lengua nacional que hispanohablantes y el de las que se describen como hablantes de las dos lenguas por igual. Incluyo a Alberto, Ana y Nelly en el primer grupo; mientras que considero que Tania, Paco, Silvia y Samuel encajan en el segundo.

Estimo que Alberto pertenece al primer grupo porque, como se puede apreciar en el Fragmento 1 a continuación, se siente más nativo del alemán que del español (líneas 2 a 4). Atribuye este sentimiento a la inseguridad que experimenta al usar este último idioma (líneas 21 y 22). Su inseguridad se debe a una serie de dificultades que enfrenta al hablarlo (línea 9), tanto en lo referente a su pronunciación (línea 10), como en lo tocante a su sintaxis (línea 14) y a su componente léxico (líneas 15 a 17). Como lo indica el hecho de que responda antes de que yo termine mi pregunta (líneas 1 y 2), Alberto no duda en describirse como más germanohablante que hispanohablante.

Fragmento 1 - Alberto me cuenta qué es lo que le cuesta en español ${ }^{6}$

1 VFM:

2 Alberto:

3

4

5 VFM:

6

7 Alberto:

8

9

10

11

12

13

14

15

16

17 ¿te concibes como un hablante[na]tivo?

[ya]

mucho más que en::

que en español

¿por qué no en español?

> ¿qué es lo que sientes ahí?<

eh::

la::

'la dificultad ${ }^{\circ}$

ya a la hora de pronunciar

eh::

a la hora de de:::

$>$ ¿cómo se dice $?<(1.2)$

$<$ hacer la frase $>$

todo el léxico

el vocabulario qu-

que siempre se me olvida=

6 Baso mis convenciones de transcripción en Jefferson (2004) y Hepburn y Bolden (2013). Las expongo en el apéndice, al final del artículo. 
Incluyo a Ana en el primer grupo porque, como se puede ver en el Fragmento 2 de abajo, considera haber vivido experiencias relevantes en el desarrollo de una persona - la pubertad (línea 13) y las primeras relaciones amorosas (línea 16) - exclusivamente en alemán (línea 17). Como lo indica luego (línea 20), se trata de acontecimientos importantes de la vida que le faltan en español. El hecho de que recalque esa carencia con una subida de volumen es indicativo de que es algo que, de cierta manera, le pesa.

Fragmento 2 - Ana me cuenta lo que le falta en español

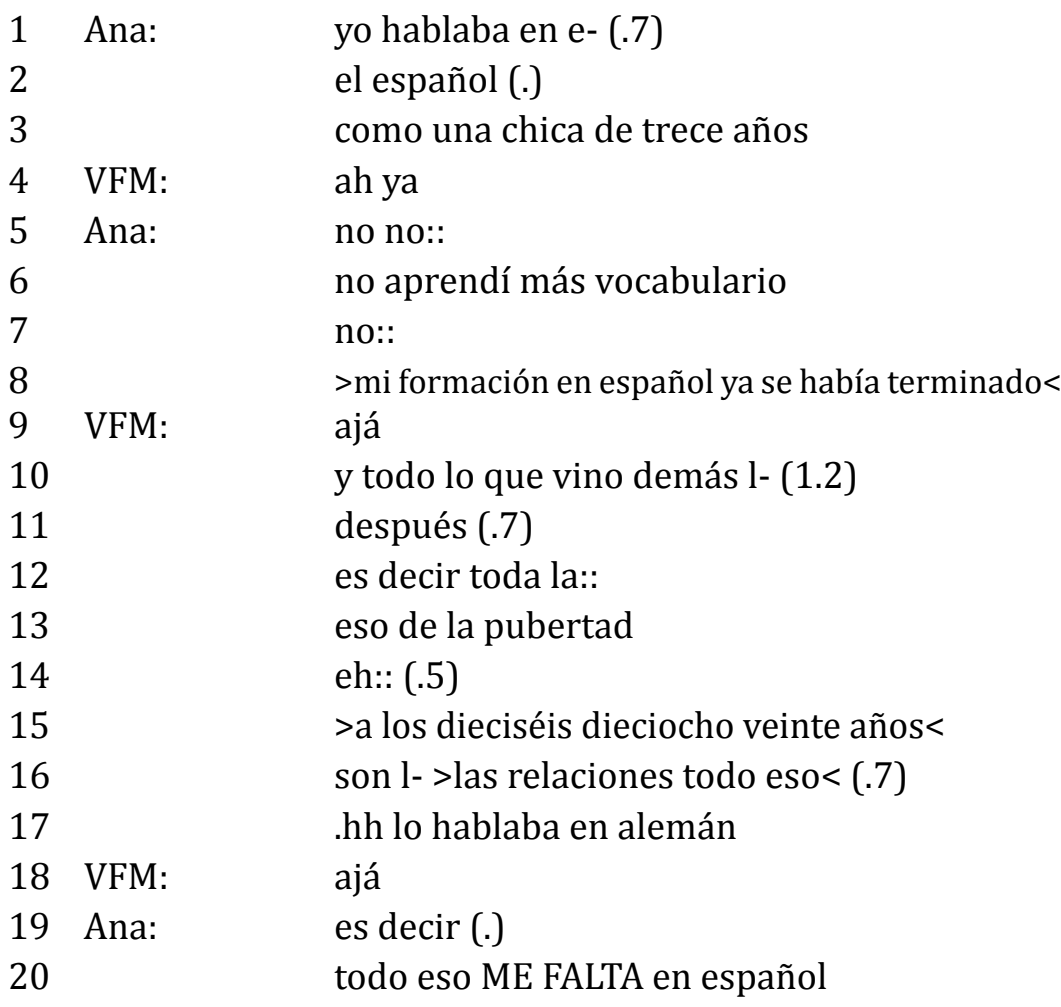

Estimo que Nelly encaja en el primer grupo debido a que, como puede verse en el Fragmento 3 a continuación, al igual que Alberto, experimenta mayores dificultades en español que en alemán (línea 3), lengua que le resulta más fácil (línea 7). Nótese que Nelly subraya la 
facilidad de uso de esta última lengua mediante el adverbio indefinido mucho ante la secuencia más fácil.

Fragmento 3 - Nelly me cuenta me cuenta cómo se siente respecto al español

$\begin{array}{lll}1 & \text { Nelly: } & \text { >pero yo no la siento como mi lengua materna< } \\ 2 & \text { VFM: } & \text { >ipor qué no la sientes como tu lengua materna?< } \\ 3 & \text { Nelly: } & \text { porque no lo hablo tan bien como el alemán } \\ 4 & \text { VFM: } & \text { ajá } \\ 5 & \text { Nelly: } & \text { es más bien por eso } \\ 6 & & \text { >porque para mí< (.) } \\ 7 & & \text { el alemán es mucho más fácil }\end{array}$

En suma, Alberto, Ana y Nelly se conciben a sí mismos más como hablantes del alemán que como hablantes del español. Como se pudo ver, tal concepción de sus personas se debe a que valoran sus niveles de confianza en español por debajo de los que tienen en alemán. La diferencia se debe o a inseguridades de naturaleza lingüística (dificultades con la pronunciación, la sintaxis y el léxico del español) o al hecho de que eventos importantes de sus vidas no ocurrieron en español, sino que en alemán.

Tonia, Paco, Silvia y Samuel, en cambio, no evocan tales diferencias en los niveles de confianza que tienen en una u otra de sus lenguas. Más bien, como se verá a continuación, juzgan tener niveles similares en las lenguas que constituyen su repertorio lingüístico. Por eso, considero que encajan en el segundo grupo.

Por ejemplo, en el Fragmento 4 de abajo, Tonia se declara perfectamente bilingüe en español y francés (línea 6). Realza sus habilidades lingüísticas con el adjetivo total, que encierra una idea de comprensión absoluta, y subiendo la voz al pronunciar detenidamente el pronombre ambas, que hace referencia a las lenguas de las que se considera como hablante perfecta.

Fragmento 4 - Tonia me cuenta que es perfectamente bilingüe

1 Tonia:

2

3

4

5

6 en la vida de todos los días (.8)

me siento::

ejem:::

eh::

$>$ no sé cómo decirlo<

total hablante de $<$ AMBAS $>$ 
Por su parte, como se puede apreciar en el Fragmento 5 a continuación, Paco observa que el hecho de sentirse hispanohablante o germanohablante está sujeto al contexto comunicativo en el que se encuentra (línea 11). Si está con hispanohablantes, se siente como un hispanohablante más (líneas 13 a 17); si se encuentra con germanohablantes, se siente como tal (líneas 18 a 21). La capacidad de Paco de concebirse como hablante de una u otra lengua en función de elementos contextuales pone de manifiesto que posee habilidades lingüísticas extraordinarias en español y en alemán.

Fragmento 5 - Paco me cuenta donde se encuentra entre el español y el alemán

$\begin{array}{lll}1 & \text { Paco: } & \text { eh::: } \\ 2 & & \text { a la mitad } \\ 3 & \text { VFM: } & =\text { ¿ cómor } ? \\ 4 & & \text { porque a veces pienso en alemán= } \\ 5 & \text { Paco: } & \text { =y a veces pienso en: (.) } \\ 6 & & \text {.hh en español } \\ 7 & & \text { ajá } \\ 8 & \text { VFM: } & \text { y eso es- eh:: } \\ 9 & \text { Paco: } & \text { <depende> (.7) } \\ 10 & & \text { depende CON QUIÉN estoy } \\ 11 & & \text { ajá } \\ 12 & \text { VFM: } & \text { si estoy más con un grupo eh:: } \\ 13 & \text { Paco: } & \text { de hispanohablantes } \\ 14 & & \text { entonces me:: } \\ 15 & & \text { me siento mas hisp- } \\ 16 & & \text { hispanohablante } \\ 17 & & >\text { pero si estoy con un grupos< } \\ 18 & & \text { con:: eh:: } \\ 19 & & \text { con hablantes del alemán (.5) } \\ 20 & & \text {-entonces es alemán } \\ 21 & & \end{array}$

Como se puede ver en el Fragmento 6 de abajo, Silvia también reporta tener competencias lingüísticas similares en español y alemán (línea 1). La particularidad de su caso es que concibe sus habilidades en ambas lenguas como un continuo (línea 2), esto es, como un todo formado por partes unidas entre sí dentro de las cuales puede desplazarse a su antojo (líneas 4 y 5). Al igual que en el caso de Paco, esta destreza revela que Silvia posee habilidades lingüísticas extraordinarias en español y en alemán. 
Fragmento 6 - Silvia me cuenta que se mueve en un continuum lingüístico

$\begin{array}{lll}1 & \text { Silvia: } & \text { me siento cómoda en ambas (.) } \\ 2 & & \text { es como un continuum }= \\ 3 & =\text { ¿sabes? } \\ 4 & & \text { a veces me muevo un poco más hacia el español } \\ 5 & & \text { a veces más hacia el alemán } \\ 6 & \text { no sé } \\ 7 & \text { es eh:: } \\ 8 & \text { es extraño }\end{array}$

Por su parte, en el Fragmento 7 a continuación, se puede observar que Samuel no advierte ninguna limitación lingüística, ni en lo que se refiere al español (líneas 7 a 9) ni en lo tocante al alemán. Esto último, aunque Samuel no lo verbaliza, se desprende del uso del adverbio tampoco (línea 8), que suele servir para negar una cosa (limitaciones en español) después de haberse negado otra (limitaciones en alemán).

Fragmento 7 - Samuel me cuenta que no siente limitaciones lingüísticas

$\begin{array}{lll}1 & \text { Samuel: } & \text { me siento bien integrado } \\ 2 & \text { VFM: } & \text { ¿sí? } \\ 3 & \text { Samuel: } & \text { sí (1.3) } \\ 4 & & \text { lingüísticamente hablando } \\ 5 & \text { VFM: } & \text { ¿y tu español? } \\ 6 & \text { Samuel: } & \text { pues @@@ } \\ 7 & & \text { @ya que estoy estudiando español@ } \\ 8 & & \text { creo que esa limitación tampoco:: (.) } \\ 9 & & \text {-exista }\end{array}$

En resumen, Tonia, Paco, Silvia y Samuel se piensan a sí mismos como hablantes de dos de las lenguas que constituyen sus repertorios lingüísticos por igual. Como se pudo ver, el hecho de que se conciban de esa manera se debe a la ausencia de limitaciones percibidas en una u otra lengua, a la capacidad de pasar de una identidad lingüística a otra en función de la identidad de sus interlocutores y a la habilidad de moverse en un continuo lingüístico que comprende el español y la lengua nacional como partes de un todo.

En cualquier caso, ninguna de las personas cuyos autorretratos aquí analizo puede considerarse como no integrada lingüísticamente a la sociedad suiza. En todos los casos, se conciben primordialmente 
como hablantes nativos de una lengua nacional (alemán o francés). En cambio, al haber desarrollado inseguridades en español, no todos se consideran hablantes naturales de esa lengua.

En lo que respecta a la autoidentificación cultural, reconocí cuatro grupos en función de las respuestas que Alberto, Ana, Tonia, Nelly, Paco, Silvia y Samuel dieron a mis interrogantes: el de las personas que se sienten más suizas que hispanas o latinas; el de las que se describen como suizas e hispanas o latinas; el de las que se conciben como una mezcla de las culturas suizas e hispanas o latinas; y el de las que no se consideran ni suizas ni hispanas o latinas.

Como puede apreciarse en el Fragmento 8 de abajo, Tonia, quien corresponde al primer perfil, se siente más suiza que española (línea 1) por cuestiones prácticas. Efectivamente, este sentimiento es provocado por el hecho de que es aquí (línea 2) - es decir, en Suiza - y no en España (líneas 6 y 7) que proyecta su futuro, en general (línea 4) y como profesional (línea 5).

Fragmento 8 - Tonia me cuenta por qué se siente más suiza

$\begin{array}{ll}1 & \text { Tonia: } \\ 2 & \\ 3 & \\ 4 & \\ 5 & \\ 6 & \\ 7 & \end{array}$

me siento más suiza

porque sí que me imagino quedándome aquí (1.5)

para to::da mi vida (.)

o viviendo aquí $=$

trabajando aquí

$>$ mientras que en España<

no me imagino hacer eso hh.

Estimo que Ana, en cambio, encaja en el grupo de las personas que se describen como suizas e hispanas o latinas. Como se puede ver en el Fragmento 9 a continuación, el uso del conector y (línea 2), que Ana enfatiza alzando considerablemente la voz, es indicativo de que suma las identidades boliviana y suiza. Nótese, eso sí, que dicha suma no implica la emergencia de una identidad nueva. Más bien, una u otra se hace más relevante en función del contexto. Con el primer uso de ahora (línea 11), Ana evoca el lugar en el que se encuentra en el momento presente en un sentido amplio: Suiza. En ese sitio, tiene relevancia su identidad helvética. En cambio, la segunda ocasión en la que usa este adverbio (línea 23), alude al lugar en el que se ubica presentemente en un sentido específico: el aula en la que está conversando conmigo, una persona de origen latino. Ahí, es su identidad boliviana la que tiene mayor trascendencia. 
Fragmento 9 - Ana me cuenta que se concibe como boliviana y suiza

$\begin{array}{lll}1 & \text { Ana: } & \begin{array}{l}\text { yo soy:: (.7) } \\ \text { <boliviana Y suiza> }\end{array} \\ 3 & \text { VFM: } & \text { ¿sí? } \\ 4 & \text { Ana: } & \begin{array}{l}\text { los dos (.) } \\ { }^{\circ} \text { sío }\end{array} \\ 5 & & \text { ¿por qué? } \\ 6 & \text { VFM: } & \text { definitivamente por:: (2) } \\ 7 & \text { Ana: } & \text { yo creo que:: } \\ 8 & & \text { por la lengua } \\ 9 & & \text { ajá } \\ 10 & \text { VFM: } & \text { ahora (1.1) } \\ 11 & \text { Ana: } & \text { yo:: pienso en alemán } \\ 12 & & \text { pienso en alemán= } \\ 13 & \text { VFM: } & \text { =sueño en alemán (.) } \\ 14 & \text { Ana: } & \text { eh:: (2.3) } \\ 15 & & \text { cuando tengo que:: } \\ 16 & & \text { cuando hablo mucho con:: } \\ 17 & & \text { cuando tengo que ver con:: } \\ 18 & & >\text { con gente digamos latina< } \\ 19 & & \text { entonces ahí sí que:: } \\ 20 & & \text { que pienso en español (2.7) } \\ 21 & & >\text { como ahora también estoy } \\ 22 & & \text { pensando en español< } \\ 23 & & \end{array}$

Como se puede apreciar en el Fragmento 10 de abajo, para Paco, la manera en la que se siente también está sujeta a elementos contextuales. En este sentido, se puede decir que, al igual que Ana, suma las identidades (en su caso, la colombiana y la suiza). La contemplación de fotos familiares lo hace identificarse con la cultura colombiana (líneas 3 a 7). En cambio, en lo profesional, se identifica con la cultura suiza (líneas 9 a 12), específicamente a causa de la cuestión de la puntualidad (líneas 14 y 15), que es un estereotipo suizo de amplia difusión.

Fragmento 10 - Paco me cuenta que se identifica con Colombia y Suiza

$\begin{array}{lll}1 & \text { Paco: } & \text { depende } \\ 2 & \text { VFM: } & \text { ajá } \\ 3 & \text { Paco: } & \text { si yo miro: (.5) }\end{array}$




$\begin{array}{lll}4 & & \text { por ejemplo: fotos } \\ 5 & & \begin{array}{l}(\text { (me muestra una foto familiar que } \\ \text { tiene en la cartera) })\end{array} \\ 6 & \text { VFM: } & \text { ajá } \\ 7 & \text { Paco: } & \text { me identifico más con colombia (.) } \\ 8 & & \text { pero no sé } \\ 9 & & \text { como trabajo } \\ 10 & & \text { trabajar y:: (.) } \\ 11 & & \text { y esas cosas } \\ 12 & & \text { es más a suizo } \\ 13 & \text { VFM: } & \text { ¿por qué? } \\ 14 & & \text { porque @me gusta ser puntual@ } \\ 15 & & \text { @y me siento pena si yo vengo unos } \\ & & \text { minutos muy tarde@ } \\ 16 & \text { VFM: } & \text { ah:: >ya ya ya< }\end{array}$

Por su parte, considero que Alberto encaja en el grupo de las personas que se conciben como una mezcla de culturas porque, como puede verse en el Fragmento 11 a continuación, es precisamente esta palabra la que utiliza (línea 4) para detallar el porqué no se identifica totalmente ni con la cultura suiza ni con la española (líneas 1 a 3).

Fragmento 11 - Alberto me cuenta que es una mezcla de lo suizo y lo español

\begin{tabular}{|c|c|c|}
\hline 1 & Alberto: & no me identifico ni con:: (.7) \\
\hline 2 & & la totalidad del suizo \\
\hline 3 & & ni con la totalidad del español (.) \\
\hline 4 & & $>0$ sea tengo esa mezcla ¿no?< \\
\hline 5 & & $y:::(5.8)$ \\
\hline & & eso \\
\hline
\end{tabular}

Estimo que Silvia también encaja en este grupo porque, como se puede apreciar en el Fragmento 12 de abajo, manifiesta poseer unos cuantos rasgos culturales suizos y otros tantos españoles (líneas 1 a 3). En ese sentido, no creo que se trate en su caso de una suma de identidades, como lo era en el caso de Ana que se vio más arriba, sino más bien de una mezcla, que, como Silvia menciona luego, le causa placer (línea 6). 
Fragmento 12 - Silvia me cuenta que le gusta su mezcla de rasgos culturales

$\begin{array}{lll}1 & \text { Silvia: } & \text { tengo::: } \\ 2 & & \text { no sé (.6) } \\ 3 & & \text { @rasgos como de las dos culturas un poco@ } \\ 4 & \text { VFM: } & \text { ajá } \\ 5 & \text { Silvia: } & \text { es (1.5) } \\ 6 & & \text { me gusta }\end{array}$

Como puede verse en el Fragmento 13 a continuación, al igual que Alberto, Samuel utiliza el vocablo mezcla (línea 2) para describirse culturalmente. Por ello, lo incluyo en el grupo de las personas que se conciben como una amalgama de culturas. Interesantemente, describe un proceso de selección voluntaria de lo que él considera ser lo mejor de cada cultura (líneas 4 a 8 ) para que emerja una nueva identidad única (línea 10).

Fragmento 13 - Samuel me cuenta que canaliza lo mejor de las dos culturas

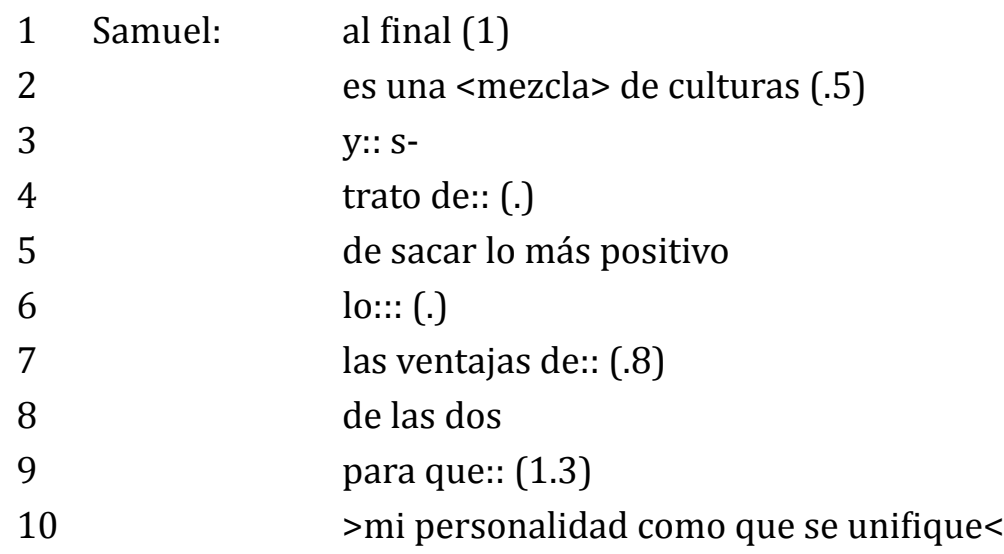

De todas las personas cuyos autorretratos aquí estudio, como se puede apreciar en el Fragmento 14 de abajo, Nelly es la única que indica no sentirse identificada ni con la cultura suiza (línea 7) ni con las culturas de sus padres (línea 9). Como indica, la razón por la cual no se identifica con la cultura helvética radica en el hecho de que, a pesar de haber nacido y crecido en Suiza, no tiene la nacionalidad (línea 6). Sin embargo, eso no significa que, por defecto, sienta afinidad con las culturas colombiana o chilena de sus padres (línea 9). Más bien, para Nelly, el tema de su identidad parece ser complicado, 
en el sentido de que no sabe con qué identidad cultural reconocerse (líneas 4 y 11). Así y todo, el hecho de que se ría al mencionarlo indica que no se trata de algo que le cause demasiada preocupación.

Fragmento 14 - Nelly me cuenta que no sabe con qué se siente identificada

$\begin{array}{lll}1 & \text { Nelly: } & \text { pero:: (.) } \\ 2 & & \text { ¿de qué nacionalidad me siento más? } \\ 3 & & \text { ni idea } \\ 4 & & \text { eso sí @que no sé@ } \\ 5 & & \text { porque no tengo tampoc- } \\ 6 & & \text { >no tengo el pasaporte suizo< } \\ 7 & & \text { no SOY suiza } \\ 8 & \text { VFM: } & \text { ajá } \\ 9 & \text { Nelly: } & \text { >pero tampoco me siento como colombiana } \\ & & \text { o chilena< } \\ 10 & & \text { entonces:: }(1.3) \\ 11 & & \text { no te sé decir @qué@ }\end{array}$

En suma, a excepción de Nelly, ninguna de las personas cuyos autorretratos constituyen la base de mi análisis puede considerarse como no integrada culturalmente a la sociedad suiza. Tonia se concibe predominantemente como una suiza por motivo de sus planes futuros en ese país. No tiene tales planes en España, el país de donde provienen sus padres. Ana y Paco se identifican tanto con la cultura suiza como con sus culturas latinoamericanas. Encima, poseen la habilidad de darle relevancia a una u otra en función de elementos contextuales. Alberto, Silvia y Samuel combinan elementos de las identidades culturales suizas e hispanas o latinas. De la mezcla, emergen nuevas identidades híbridas que incluyen (los mejores) elementos de las que les sirven de base. En el caso de Nelly, la falta de identificación con la cultura local parece ser consecuencia de una falta de reconocimiento de esta cultura hacia su persona. Como se mencionó más arriba, esta se debe al hecho de que Suiza no tiene derecho del suelo.

En lo tocante a los factores que podrían haber tenido cierta incidencia en la autoidentificación de las personas, hallé que estos solo tienen cierta relevancia en lo que concierne a la manera en la que las personas se reconocen culturalmente, mas no lingüísticamente. En concreto, las dos personas que suman las identidades suiza e hispana o latina y que poseen la habilidad de darle más relevancia a la una o a la otra en función del contexto -esto es, Ana y Paco- nacieron en países hispanohablantes y tienen progenitores mixtos. Existe 
pues la posibilidad de que tanto su experiencia en estos países de habla hispana como el modelo de sus progenitores les haya facilitado el desarrollo de esta destreza sin parangón. Para el resto de los casos, es difícil observar regularidades. En el caso de las personas que se sienten más suizas que hispanas o latinas, solo cuento con la autoidentificación de Tonia. Lo mismo sucede en lo que atañe a las personas que no se reconocen ni como suizas ni como hispanas o latinas, donde cuento únicamente con la autoidentificación de Nelly. En cuanto a Alberto, Silvia y Samuel, que son las personas que se conciben como una mezcla de las culturas suizas e hispanas o latinas, resulta que tienen antecedentes bastante distintos. Mientras que dos nacieron en Suiza, la otra nació en un país de habla hispana. Asimismo, mientras que una tiene progenitores mixtos, los de las dos otras nacieron en países hispanohablantes.

\section{Discusión y cierre}

La evidencia presentada en este estudio permite poner a pruebay rectificar, con datos empíricos, dos tendencias discursivas observadas a nivel nacional suizo y a nivel internacional en los llamados países receptores de migrantes. Como se vio más arriba, estas tendencias consisten en concebir a los migrantes y sus descendientes como un sector demográfico que no se integra ni lingüística ni culturalmente a la población local y, consecuentemente, en imaginarlos como una población homogénea en sus características lingüístico-culturales. La evidencia obtenida sobre la base de las descripciones que Alberto, Ana, Tonia, Nelly, Paco, Silvia y Samuel hicieron de sus personas muestra que esto no es para nada así. En lo tocante a la lengua, como se pudo ver, ninguna de estas personas se describe como alguien que no habla uno de los idiomas nacionales. Es más, nadie se presenta siquiera como un hablante con limitaciones en uno de estos idiomas. Lo contrario es cierto, en el sentido de que algunos hablantes mencionan sentir inseguridades o tener carencias importantes en español, la lengua que han adquirido en casa con sus progenitores o en el país en el que pasaron parte de su infancia. En lo que concierne al aspecto cultural, se vio que, con la excepción de Nelly, todas estas personas sienten haber integrado elementos de la llamada cultura local a su identidad. En el caso de Tonia, estas marcas de identidad local son más relevantes que las de su identidad cultural española. En lo que concierne a Ana y a Paco, se pudo ver que poseen la habilidad de canalizar una u otra de sus identidades cuando, en función del contexto comunicativo, esta cobra relevancia. En cuanto a Alberto, Silvia y Samuel, pudo constatarse que se han forjado una identidad híbrida que comprende elementos de su identidad cultural suiza y elementos de 
sus identidades culturales españolas o latinoamericanas. Así pues, los autorretratos de estas siete personas permiten resaltar el hecho de que ni sus prácticas lingüísticas ni sus identidades culturales pueden reducirse a simples dicotomías del tipo hablante o no de una lengua o persona con o sin afiliaciones a una cultura en particular. De igual forma, permiten relativizar los conceptos de bilingüismo y biculturalismo, tal como se entienden popularmente, para concebirlos más bien como fenómenos escalares (Toribio, 2011) y contextualmente adaptables (Schwartz y Unger, 2010). Por otra parte, los autorretratos aquí presentados ponen de manifiesto la diversidad inherente que existe dentro de colectivos de personas que comúnmente se entienden como homogéneos. Asimismo, ya sea por la habilidad de darle prevalencia a la identidad suiza sobre la española por motivo de planear su vida en Suiza (Tonia), por la destreza de moverse en un continuo lingüístico que va del español al alemán (Silvia) o por el arte de mezclar lo suizo y lo latino para que emerja una identidad propia (Samuel), estos autorretratos ayudan a echar luz sobre la conciencia y capacidad de control que estas personas tienen sobre sus prácticas lingüístico-culturales. Esto es relevante porque, como señala Zentella (2003:52) con referencia a las poblaciones latinas asentadas en los Estados Unidos, este sector de la población no suele concebirse como un colectivo con capacidad de control sobre sus prácticas lingüístico-culturales; más bien, suele imaginárselos como un sector demográfico que necesita ser controlado en este y otros aspectos por fuerzas que se creen superiores.

Igualmente, los autorretratos presentados en este estudio permiten verificar, con un enfoque basado en el discurso, los datos censales presentados por de Flaugergues (2016), así como expandir los hallazgos de Diez del Corral Areta (por aparecer) a la población migrante de la segunda generación. Como ya se vio, los datos que de Flaugergues (2016) examina indican que, en Suiza, más de $90 \%$ de la población migrante de la segunda generación ha incorporado una de las lenguas oficiales del país a su repertorio lingüístico, usándola con regularidad. Por su parte, los datos de Diez del Corral Areta (por aparecer) ponen de manifiesto que la población migrante de la primera generación se ha integrado a su entorno suizo tanto lingüística como culturalmente. Lo observado en el presente estudio está en línea con lo presentado en los trabajos de estas autoras.

En sí, mis hallazgos no son sorprendentes. Más bien, lo inverosímil es la prevalencia de los discursos que delinean a la población migrante como un sector demográfico que tiene dificultades para integrarse lingüística y culturalmente a la sociedad local aun cuando datos censales nacionales como los examinados por de Flaugergues (2016) y 
estudios como el de Diez del Corral Areta (por aparecer) los desmienten. A ello se suma el hecho de que estudios realizados en otros contextos que el suizo también ponen de relieve que estos discursos no tienen sustento en hechos probados. Calvi (2015), por ejemplo, estudia la autoidentificación étnica en entrevistas a migrantes hispanoamericanos en Italia. Centra parte de su análisis en la manera en la que las personas entrevistadas miden su propio grado de integración a la sociedad de acogida. Entre otras cosas, la autora halla que, con el paso del tiempo, algunas de estas personas se van sintiendo más italianas que hispanoamericanas (p.125). También, nota que no perciben el mantenimiento de elementos de la identidad lingüístico-cultural de procedencia y la integración lingüística a la sociedad de acogida como fenómenos incompatibles (p.121). Con referencia al grado de integración lingüística de latinos nacidos y criados en los Estados Unidos, existe una extensa bibliografía que observa en ese sector de la población una propensión a abandonar progresivamente el español a favor del inglés, la lengua que, por consenso nacional se percibe como local (véanse, por ejemplo, Hudson, Hernández-Chávez y Bills, 1995; Porcel, 2006; Zentella, 1997). En suma, la integración lingüístico-cultural de las poblaciones migrantes a la sociedad del lugar de destino parece ser la regla general y no la excepción.

Aparte de las anteriores implicaciones teóricas de los hallazgos del presente trabajo de investigación, también pueden discutirse algunas implicaciones prácticas. Estas se dirigen hacia la identificación de condiciones que permitan neutralizar la difusión y la aceptación de discursos reduccionistas y simplificadores sobre las poblaciones migrantes tales como los discutidos en la introducción de este artículo. Las observaciones de mi estudio y de otros trabajos de investigación que dan relevancia al carácter flexible de las identidades lingüístico-culturales de las personas las establecen como un conjunto de rasgos que pueden hacerse más o menos relevantes en función de elementos contextuales. En este sentido, Suiza, que se encuentra entre los llamados países receptores de migrantes y, en realidad, todos los espacios constitucionales y/o legales a los que llegan a vivir personas de otro lugar deben hacer esfuerzos considerables con el objetivo de mejorar las relaciones entre este sector de la población y la llamada población local. Concretamente, dadas las nuevas realidades que emergen de los movimientos poblacionales contemporáneos, es preciso que estos países y/o espacios se alejen de conceptualizaciones fijas e inmóviles de las identidades culturales de la población migrante; revoquen políticas migratorias que la conciba con ese punto de vista; y dejen de divulgar los discursos simplificadores que emergen de estas conceptualizaciones y de estas políticas. Como lo señala Augé (2009:85), las antiguas concepciones 
binarias de la frontera, la cultura y la identidad son el fermento de enfrentamientos y violencias entre poblaciones con ascendientes migrantes y las llamadas poblaciones locales. Así pues, tal y como lo indica el autor (p. 91), es necesario revalorizar a los individuos que provienen de la migración y sus biografías transculturales, puesto que, debido a su orientación hacia distintas identidades culturales, son quienes tienen la capacidad de no renunciar a ninguna de ellas. De este modo, son quienes pueden incidir en que se reconozca cada uno de los componentes de estas identidades en su justo valor.

Apéndice: convenciones de transcripción

\begin{tabular}{|c|c|}
\hline Símbolo & Uso \\
\hline $\begin{array}{l}\text { texto (\# de segundos) } \\
\text { texto }\end{array}$ & Indica la duración de una pausa en segundos. \\
\hline texto (.) texto & $\begin{array}{l}\text { Indica una pausa con una duración menor a } .2 \\
\text { segundos. }\end{array}$ \\
\hline \multirow{3}{*}{ [texto] } & $\begin{array}{l}\text { Indica el principio y el final de un solapamiento. } \\
\text { Por ejemplo: }\end{array}$ \\
\hline & ¿quieres comer [algo]? \\
\hline & [quiero] comer helado \\
\hline \multirow{4}{*}{ texto $=$ texto } & $\begin{array}{l}\text { Indica un cambio de unidad de entonación sin } \\
\text { interrupción. Por ejemplo: }\end{array}$ \\
\hline & fui al garaje= \\
\hline & $=e l$ que me recomendaste \\
\hline & me gustó \\
\hline texto & Precede un tono ascendente. \\
\hline -texto & Precede un tono descendente. \\
\hline ¿texto? & Marca una entonación de pregunta. \\
\hline$>$ texto $<$ & $\begin{array}{l}\text { Indica que se dijo algo de una manera más rápi- } \\
\text { da de lo habitual. }\end{array}$ \\
\hline$<$ texto $>$ & $\begin{array}{l}\text { Indica que se dijo algo de una manera más lenta } \\
\text { de lo habitual. }\end{array}$ \\
\hline
\end{tabular}


Marca un alargamiento del sonido anterior. A mayor número de dos puntos, mayor el alargamiento. Por ejemplo:

texto:::

creo que es algo:::

fantá:stico

\begin{tabular}{ll}
\hline hh. & Exhalación audible. \\
\hline hh & Inhalación audible. \\
\hline${ }^{\circ}$ texto $^{\circ}$ & Indica que se susurró algo. \\
\hline @@ & $\begin{array}{l}\text { Indica risa. El número de arrobas marca la dura- } \\
\text { ción de la risa en segundos. }\end{array}$ \\
\hline @texto@ & Indica que el texto entre arrobas se dijo riendo. \\
\hline tex- & Marca una interrupción brusca. \\
\hline TEXT0 & Indica que se dijo algo con volumen de voz alto. \\
\hline & $\begin{array}{l}\text { El texto entre paréntesis dobles indica activi- } \\
\text { dades no-verbales. Por ejemplo: }\end{array}$ \\
((texto)) & (rascándose la cabeza)) no sé \\
\hline
\end{tabular}

\section{Bibliografía}

Augé, M. (2009). Pour une anthropologie de la mobilité. Paris : Payot \& Rivages.

Bircher: (1998). Étrangers résidant en Suisse. Promotion d'une langue nationale. Berne : Le Parlement suisse. Revisado el 21 de mayo de 2018 desde Internet: https://www.parlament.ch/fr/ratsbetrieb/suche-curia-vista/geschaeft?AffairId=19983465

Blommaert, J. (2005). Discourse. Cambridge: Cambridge University Press.

Calvi, M. V. (2015). Etiquetas étnicas e identidad en entrevistas a inmigrantes hispanoamericanos en Italia. En: L. Mariottini (Ed.). Identità e discorsi. Studi offerti a Franca Orletti. (pp. 111-130). Roma: Roma TrE-Press. 
De Flaugergues, A. (2016). Pratiques linguistiques en Suisse: premiers résultats de l'enquête sur la langue, la religion et la culture 2014. Neuchâtel : Office fédéral de la statistique. Revisado el 22 de mayo de 2018 desde Internet : https://www.bfs. admin.ch/bfs/fr/home/statistiques/population.assetdetail.1000171.html

Diez del Corral Areta, E. (por aparecer). Me siento muy poquititico suiza: identificación bilingüe y bicultural en inmigrantes colombianos de la Suiza francófona. Lengua y migración, 10, (2).

Duchêne, A., y Del Percio, A. (2014). Economic capitalization of linguistic diversity: Swiss multilingualism as a national profit? En: J. W. Unger, M. Krzyzanowski, y R. Wodak (Eds.). Multilingual encounters in Europe's institutional spaces. (pp. 75-101). London et al.: Bloomsbury.

Fernández-Suárez, B. (2017). The design of migrant integration policies in Spain: discourses and social actors. Social Inclusion, 5(1), 117-125.

Flubacher, M. (2013). Language(s) as the key to integration? The ideological role of diglossia in the German-speaking region of Switzerland. En: E. Barát: Studer, y J. Nekvapil (Eds.). Ideological conceptualization of language: discourses of linguistic diversity. (pp. 171-192). Frankfurt am Main: Peter Lang.

García, 0. (1993). From Goya portraits to Goya beans: elite traditions and popular streams in U.S. language policy. Southwest Journal of Linguistics, 12, 69-86.

Grosjean, F. (2008). The bicultural person: a short introduction. En: F. Grosjean (Ed.). Studying bilinguals. (pp. 213-220). Oxford y New York: Oxford University Press.

Hepburn, A., y Bolden, G. B. (2013). The conversation analytic approach to transcription. En: J. Sidnell y T. Stivers (Eds.). The handbook of conversation analysis. (pp. 57-76). Malden: Wiley-Blackwell.

Hudson, A., Hernández-Chávez, E., y Bills, G. (1995). The many faces of language maintenance: Spanish language claiming in five Southwestern states. En: C. Silva-Corvalán (Ed.). Spanish in four continents: studies in language contact and bilingualism. (pp. 165-183). Washington, DC: Georgetown University Press.

Hymes, D. (1996). Ethnography, linguistics, narrative inequality: toward and understanding of voice. London: Taylor \& Francis. 
Irvine, J. T., y Gal, S. (2000). Language ideology and linguistic differentiation. En: P. Kroskrity (Ed.). Regimes of language. (pp. 3583). Santa Fe: School of American Research Press.

Jefferson, G. (2004). Glossary of transcript symbols with an introduction. En: G. H. Lerner (Ed.). Conversation analysis: studies from the first generation. (pp.13-31). Amsterdam: John Benjamins.

Johnstone, B. (2008). Discourse analysis. (2a ed.). Malden et al.: Blackwell.

Ley Federal de Extranjería. (2005). Loi fédérale sur les étrangers (LEtr). Berne : Le Conseil fédéral. Revisado el 21 de mayo de 2018 desde Internet: https://www.admin.ch/opc/fr/classified-compilation/20020232/index.html

Liebscher, G., y Dailey-0'Cain, J. (2009). Language attitudes in interaction. Journal of Sociolinguistics, 13(2), 195-222.

Lüdi, G., y Werlen, I. (2005). Le paysage linguistique en Suisse. Neuchâtel : Office fédéral de la statistique. Revisado el 22 de mayo de 2018 desde Internet: https://www.bfs.admin.ch/ bfs/fr/home/statistiques/population/langues-religions/ langues.assetdetail.342098.html

Porcel, J. (2006). The paradox of Spanish among Miami Cubans. Journal of Sociolinguistics, 10(1), 93-110.

Rumbaut, R. G. (2004). Ages, life stages, and generational cohorts: decomposing the immigrant first and second generations in the United States. International Migration Review, 38(3), 1160-1205.

Sánchez, R. (2016). Edificando espacios comunicativo-identitarios. Términos de autoidentificación en la prensa hispanófona neoyorquina durante la primera mitad del siglo XX. En: Y. Bürki y H. Partzsch (Eds.). Redes de comunicación: estudios sobre la prensa en el mundo hispanohablante. (pp. 249-279). Berlin: Frank \& Timme.

Schwartz, S. J., y Unger, J. B. (2010). Biculturalism and context: what is biculturalism and when is it adaptive? Human Development, 53(1), 26-32.

Toribio, A. J. (2011). Spanish English code-switching among US Latinos. International Journal of the Sociology of Language, 158, 89-119.

Wessendorf, S. (2013). Second-generation transnationalism and roots migration: cross-border lives. London y New York: Routledge. 
Yeung, S. (2016). From cultural distance to skills and deficits: "expatriates," "migrants" and Swiss integration policy. Multilingua, 35(6), 723-746.

Zentella, A. C. (1997). Growing up bilingual: Puerto Rican children in New York. Oxford: Blackwell.

Zentella, A. C. (2003). “José, can you see?”: Latin@ responses to racist discourse. En: D. Sommer (Ed.). Bilingual games: some literary investigations. (pp. 51-66). New York y Basingstoke: Palgrave. 